This item was submitted to Loughborough's Research Repository by the author.

Items in Figshare are protected by copyright, with all rights reserved, unless otherwise indicated.

\title{
Sulfimidation of thioether groups - a versatile method for modifying and linking thia/oxa crowns
}

PLEASE CITE THE PUBLISHED VERSION

http://dx.doi.org/10.1039/b802903b

\section{PUBLISHER}

(c) The Royal Society of Chemistry

\section{VERSION}

AM (Accepted Manuscript)

\section{PUBLISHER STATEMENT}

This work is made available according to the conditions of the Creative Commons Attribution-NonCommercialNoDerivatives 4.0 International (CC BY-NC-ND 4.0) licence. Full details of this licence are available at: https://creativecommons.org/licenses/by-nc-nd/4.0/

\section{LICENCE}

CC BY-NC-ND 4.0

\section{REPOSITORY RECORD}

Elsegood, Mark R.J., Paul F. Kelly, Gillian Reid, Alexandra M.Z. Slawin, and Paul M. Staniland. 2019.

"Sulfimidation of Thioether Groups - a Versatile Method for Modifying and Linking Thia/oxa Crowns". figshare. https://hdl.handle.net/2134/15614. 


\title{
Sulfimidation of thioether groups - a versatile method for modifying and linking thia/oxa crowns
}

\author{
Mark R. J. Elsegood ${ }^{\mathrm{a}}$, Paul F. Kelly ${ }^{\mathrm{a}^{*}}$, Gillian Reid ${ }^{\mathrm{b}^{*}}$, Alexandra M. Z. Slawin ${ }^{\mathrm{c}}$ and Paul M. Staniland \\ Received (in XXX, XXX) 1st January 2007, Accepted 1st January 2007 \\ ${ }_{5}$ First published on the web 1st January 2007 \\ DOI: 10.1039/b000000x
}

Reaction of the mixed thioether/ether crowns [9] $\mathrm{aneO}_{2} \mathrm{~S} \mathrm{1}$, [12] $\mathrm{aneO}_{3} \mathrm{~S} 2$ and [18]aneO ${ }_{4} \mathrm{~S}_{2} \mathbf{3}$ with one mol. equiv. of the aminating agent $\mathrm{MSH}$ (o-mesitylsulfonylhydroxylamine) in $\mathrm{Et}_{2} \mathrm{O}$ results in the formation of the mono-sulfimidated systems $\left\{[9] \mathrm{aneO}_{2}\left(\mathrm{~S}=\mathrm{NH}_{2}\right)\right\}^{+} \mathbf{1 a},\left\{[12] \mathrm{aneO}_{3}\left(\mathrm{~S}=\mathrm{NH}_{2}\right)\right\}^{+} \mathbf{2 a}$

10 and $\left\{[18] \mathrm{aneO}_{4} \mathrm{~S}\left(\mathrm{~S}=\mathrm{NH}_{2}\right)\right\}^{+}$3a, while using two mol. equivalents of $\mathrm{MSH}$ with $\mathbf{3}$ gives the disulfimidium cation $\left\{[18] \mathrm{aneO}_{4}\left(\mathrm{~S}=\mathrm{NH}_{2}\right)_{2}\right\}^{2+} \mathbf{3 b}$. All of these species have been isolated in good yields as the $\left[\mathrm{mesSO}_{3}\right]^{-}$(mes $=2,4,6-\mathrm{Me}_{3} \mathrm{C}_{6} \mathrm{H}_{2}$ ) salts and can be readily converted readily to the $\left[\mathrm{BPh}_{4}\right]^{-}$salts by metathesis with $\mathrm{Na}\left[\mathrm{BPh}_{4}\right]$. Treatment of $\mathbf{1 a}$ or $\mathbf{2 a}$ with lithium diisopropylamide (LDA) and N-bromosuccinimide (NBS) at $-78^{\circ} \mathrm{C}$, followed by addition of a further equivalent of 15 the parent thia/oza crown, gives monocationic N-bridged sulfimide bicyclic compounds (4 and $\mathbf{5}$ respectively), in which the crowns are linked by the sulfimidic nitrogen. Reaction of 3a with LDA and NBS leads to formation of the $\left\{\left([18] \mathrm{aneO}_{4} \mathrm{~S}_{2}\right) \mathrm{N}\right\}^{+}$cation 6 which exhibits an intramolecular $\mathrm{S}-\mathrm{N}-\mathrm{S}$ bridge. Crystallographic studies on representative examples of each compound type are described, together with their spectroscopic properties.

\section{${ }_{20}$ Introduction}

The importance of crown ether systems systems to all areas of the chemical community continues to grow. ${ }^{1}$ Their significance stems from the range of properties they display (not the least of which is their ability to encapsulate metal 25 cations) and has inspired the preparation of a wide range of examples of derivatised versions of archetypal crowns such as 18-crown-6. These are generally prepared through modification of the carbon backbones via incorporation of functionalised groups into the macrocycle. Although this can 30 involve a range of different groups, and the resulting products can, in turn, react to derivatise the systems still further, in all such cases the variation upon the basic crown ether unit is brought about by modification to the carbon backbone prior to cyclisation. This is because the inertness of both the ${ }_{35}$ backbone, and of the oxygen atoms, precludes derivatisation post ring closure.

Mixed thia/oxa crowns have been studied widely, again, mainly due to their ability to encapsulate metal cations. Thus the transition metal chemistry of [18]ane $\mathrm{O}_{5} \mathrm{~S}$ and [18]ane $\mathrm{O}_{4} \mathrm{~S}_{2}$ 40 (3) (and, indeed, of the the Se/O and $\mathrm{Te} / \mathrm{O}$ analogues of the former) has been explored extensively by one of the authors $(\mathrm{GR}){ }^{2}$ In contrast to the crowns containing only oxa-ether units, these mixed systems do have the potential to undergo derivatisation thanks to the presence of the more reactive thia45 ether centre(s). Despite this clear possibility, post ringclosure modification of these types of macrocycles is still poorly documented. In one example, Dann et $a l^{3}$ reported oxidised versions of $\mathbf{3}$, formed by reacting the 'free' macrocycle with Chloramine- $\mathrm{T}$ and with hydrogen peroxide, 50 generating $[18]$ aneO $_{4}(\mathrm{~S}=\mathrm{NTs})_{2}$ and $\quad[18] \mathrm{aneO}_{4}(\mathrm{~S}=\mathrm{O})_{2}$ respectively. Some subsequent work on this sulfoxide was reported, ${ }^{4}$ as were studies into examples of thia-crowns bearing $\mathrm{S}=\mathrm{O}$ units, ${ }^{5}$ but until a recent preliminary report on our part, no examples were known of systems wherein this 55 methodology had been used to extend functional derivatisation of crowns.

In that preliminary report we showed that reaction of [18] ane $\mathrm{O}_{5} \mathrm{~S}$ with the aminating agent $\mathrm{MSH}$ resulted in the formation of the sulfimidium cation, $\left\{[18] \mathrm{aneO}_{5}\left(\mathrm{~S}=\mathrm{NH}_{2}\right\}^{+}\right.$ 60 (Scheme 1). ${ }^{6} \quad$ Deprotonation and bromination, using, consecutively, lithium diisopropylamide (LDA) and $\mathrm{N}$ bromosuccinimide (NBS), allowed the in situ generation of [18] ane $\mathrm{O}_{5}(\mathrm{~S}=\mathrm{NBr})$. The known temperature-sensitive nature of dialkylsulfimides necessitated the one-pot reaction be 65 carried out at low temperature $\left(-78^{\circ} \mathrm{C}\right)$ and the product reacted immediately, in this case with the parent crown. This resulted in the linked crown system $\left[\left(\left\{[18] a n e \mathrm{O}_{5} \mathrm{~S}\right\}_{2} \mathrm{~N}\right)\right]^{+}$, in which the two crowns are joined via a sulfimide nitrogen. Despite the additional charge on the linked crowns imparted 70 by the $\mathrm{S}-\mathrm{N}-\mathrm{S}$ bridge, importantly we were also able to demonstrate that the molecule was still able to accommodate a sodium cation bound via the ether $\mathrm{O}$ atoms.

The aforementioned report thus confirmed the viability of an entirely new class of reaction within crown ether 75 chemistry, namely significant post ring-closure derivatisation. Here we demonstrate that this technique is applicable to a range of other ring systems and, in addition, that within the dithiacrown [18]ane $\mathrm{O}_{4} \mathrm{~S}_{2}$ intramolecular sulfimidation is also possible.

\section{${ }_{80}$ Experimental}

\section{Instrumentation and conditions}

Infrared spectra were recorded as pressed $\mathrm{KBr}$ pellets over the range $4000-200 \mathrm{~cm}^{-1}$ on a Perkin-Elmer System 2000 Fourier- 
transform spectrometer, ${ }^{1} \mathrm{H}(400 \mathrm{MHz})$ and ${ }^{13} \mathrm{C}\left\{{ }^{1} \mathrm{H}\right\}$ NMR spectra $(100 \mathrm{MHz})$ on a Bruker DPX-400 FT spectrometer with chemical shifts in ppm to high frequency of $\mathrm{SiMe}_{4}$ and coupling constants ( $\mathrm{J}$ ) in Hz. Elemental analyses (Perkin5 Elmer 2400 CHN or Exeter Analytical, Inc. CE-440 Elemental Analyzers) were performed by the Loughborough University Analytical Service within the Department of Chemistry; FAB mass spectra were recorded on a JEOL SX102 mass spectrometer, $\mathrm{ES}^{+}$and $\mathrm{ES}^{-}$mass spectra (MeCN solution) 10 were recorded using a VG Biotech platform or carried out by the EPSRC Mass Spectrometry Service at the University of Wales, Swansea.

All reactions were performed under an atmosphere of dry nitrogen using standard Schlenk techniques and appropriately 15 dried solvents. [9] ane $\mathrm{O}_{2} \mathrm{~S}$ and [18] $\mathrm{aneO}_{4} \mathrm{~S}_{2}$ were prepared via the literature procedure, and [12] $\mathrm{aneO}_{3} \mathrm{~S}$ was prepared using a similar method. ${ }^{7} \underline{O}$-mesitylsulfonylhydroxylamine (MSH) was prepared by the literature route, ${ }^{\mathbf{8}}$ while lithium diisopropylamide (LDA) and N-bromosuccinimide (NBS) 20 were used as received (Aldrich).

SAFETY NOTE!: MSH can spontaneously (and unpredictably) decompose, resulting in an explosive pressure change. Samples should be kept in small amounts in loosely sealed containers, thereby minimising the risks should 25 decomposition occur.

\section{Preparations}

\section{Reactions of [9]ane $\mathrm{O}_{2} \mathrm{~S}(1)$}

30 [1a][mesSO ${ }_{3}$ ]: [9]ane ${ }_{2} \mathrm{~S}(1.0 \mathrm{~g}, 6.75 \mathrm{mmol})$ was dissolved in $\mathrm{Et}_{2} \mathrm{O}\left(15 \mathrm{~cm}^{3}\right)$, and MSH (1.82 g, $\left.8.43 \mathrm{mmol}\right)$ added as a solid in several portions. The mixture was stirred overnight, after which the white solid produced was filtered, washed with further $\mathrm{Et}_{2} \mathrm{O}\left(2 \times 25 \mathrm{~cm}^{3}\right)$ and dried in vacuo. Yield: $2.13 \mathrm{~g}$ 35 (87\%). Found: C, 49.39; $\mathrm{H}, \quad 6.95 ; \mathrm{N}, \quad 3.48$. $\mathrm{C}_{15} \mathrm{H}_{25} \mathrm{O}_{4} \mathrm{~S}_{3} \mathrm{~N}$.0.5Et ${ }_{2} \mathrm{O}$ requires $\mathrm{C}, 49.02 ; \mathrm{H}, 7.26 ; \mathrm{N}, 3.36 \%$. IR $v_{\max } / \mathrm{cm}^{-1}$ : 3195, 3026, 1603, 1451, 1184, 1012, 845, 746, 682, 580, 549. ${ }^{1} \mathrm{H}$ NMR $\left(\mathrm{CDCl}_{3}\right): \delta_{\mathrm{H}}=6.72(\mathrm{~s}, 2 \mathrm{H}, \mathrm{Ar}-\mathrm{H})$, 6.30 (br s, 2H, S=NH $)$, 3.30-4.11 ( $\left.m, 12 \mathrm{H}, \mathrm{CH}_{2}\right), 2.56(\mathrm{~s}, 6 \mathrm{H}$, $\left.402 \times \mathrm{CH}_{3}\right), 2.14\left(\mathrm{~s}, 3 \mathrm{H}, 1 \times \mathrm{CH}_{3}\right) .{ }^{13} \mathrm{C}\left\{{ }^{1} \mathrm{H}\right\} \mathrm{NMR}\left(\mathrm{CDCl}_{3}\right) \delta_{\mathrm{C}}=$ 139.0, 137.2 (quaternary $\mathrm{Ar}-\mathrm{C}$ ), 130.8 (2 x Ar-CH), 71.3, $70.3\left(\mathrm{OCH}_{2}\right), 48.3\left(\mathrm{CH}_{2} \mathrm{SNH}_{2}\right), 23.0\left(2 \times \mathrm{CH}_{3}\right), 20.8(1 \mathrm{x}$ $\mathrm{CH}_{3}$ ). FAB MS (3-nitrobenzyl alcohol): $\mathrm{m} / \mathrm{z}=164.0$ $\left(\mathrm{C}_{6} \mathrm{H}_{14} \mathrm{~S}_{2} \mathrm{ON}\right.$ requires 164.2).

45 [1a][BPh $\mathbf{4}$ : : [1a] $\left[\mathrm{mesSO}_{3}\right](0.52 \mathrm{~g}, 1.43 \mathrm{mmol})$ was dissolved in $\mathrm{MeOH}\left(10 \mathrm{~cm}^{3}\right) . \mathrm{Na}\left[\mathrm{BPh}_{4}\right](0.61 \mathrm{~g}, 1.79 \mathrm{mmol})$ was dissolved separately in $\mathrm{MeOH}\left(5 \mathrm{~cm}^{3}\right)$ and added dropwise to the stirring crown solution. After stirring for $3 \mathrm{~h}$, the resulting white precipitate was filtered, the solid recrystallised from ${ }_{50} \mathrm{CH}_{2} \mathrm{Cl}_{2} / \mathrm{Et}_{2} \mathrm{O}$, refiltered and dried in vacuo. Yield: $0.59 \mathrm{~g}$ (85\%). Found: C, 71.34; H, 6.68; N, 2.62. $\mathrm{C}_{30} \mathrm{H}_{34} \mathrm{O}_{2}$ SNB.0.25CH $\mathrm{Cl}_{2}$ (504.7) requires C, 71.99; $\mathrm{H}, 6.89$; N, 2.78\%.

IR $v_{\max } / \mathrm{cm}^{-1}: 3216,3055,3002,1579,1479,1427,1391$, 55 1266, 1152, 1086, 851, 739, 708, 626, 602. ${ }^{1} \mathrm{H}$ NMR $\left(d^{6}-\right.$ DMSO) $\delta_{\mathrm{H}}=7.21-6.82$ (br $m, 20 \mathrm{H}, \mathrm{BPh}_{4} \mathrm{Ar}-\mathrm{CH}$ ), 3.39-4.15 $\left(m, 12 \mathrm{H}, \mathrm{CH}_{2}\right) .{ }^{13} \mathrm{C}\left\{{ }^{1} \mathrm{H}\right\} \mathrm{NMR}\left(\mathrm{d}^{6}\right.$-DMSO) $\delta_{\mathrm{C}}=163.5(1: 1: 1: 1$ q, $\mathrm{BPh}_{4}$ ipso-C), 135.5, 125.3, $121.5(\mathrm{Ar}-\mathrm{C}), 70.1,64.9$ $\left(\mathrm{OCH}_{2}\right), 46.2\left(\mathrm{CH}_{2} \mathrm{SNH}_{2}\right)$. FAB MS (3-nitrobenzyl alcohol): $60 \mathrm{~m} / \mathrm{z}=164.0\left(\mathrm{C}_{6} \mathrm{H}_{14} \mathrm{~S}_{2} \mathrm{ON}\right.$ requires 164.2).

\section{Reactions of [12]ane $\mathrm{O}_{3} \mathrm{~S}(2)$}

These were prepared using an anlogous method to those of $\mathbf{1}$.

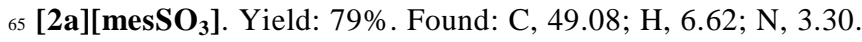
$\mathrm{C}_{15} \mathrm{H}_{25} \mathrm{O}_{4} \mathrm{~S}_{3} \mathrm{~N}$ requires $\mathrm{C}, 50.10 ; \mathrm{H}, 7.17 ; \mathrm{N}, 3.44 \%$. IR $v_{\max } / \mathrm{cm}^{-1}:$ 3180, 3044, 2964, 2924, 2853, 1457, 1376, 1210, 1179, 1087, 1014, 918, 858, 681. ${ }^{1} \mathrm{H}$ NMR (d $\left.-\mathrm{DMSO}\right) \delta_{\mathrm{H}}=$ $6.78(\mathrm{~s}, 2 \mathrm{H}, \mathrm{Ar}-\mathrm{H}), 6.04\left(\mathrm{~s}, 2 \mathrm{H}, \mathrm{NH}_{2}\right), 3.37-4.00(\mathrm{~m}, 16 \mathrm{H}$, ${ }_{70} \mathrm{CH}_{2}$ ), 2.52 (s, 6H, $\left.\mathrm{CH}_{3}\right), 2.18\left(\mathrm{~s}, 3 \mathrm{H}, \mathrm{CH}_{3}\right) .{ }^{13} \mathrm{C}\left\{{ }^{1} \mathrm{H}\right\} \mathrm{NMR}$ $\left(\mathrm{d}^{6}-\mathrm{DMSO}\right) \delta_{\mathrm{C}}=142.2,136.6,135.9$ (quaternary $\mathrm{Ar}-\mathrm{C}$ ), $129.9(\mathrm{Ar}-\mathrm{CH}), 70.0,69.6,63.8\left(\mathrm{OCH}_{2}\right), 49.0\left(\mathrm{CH}_{2} \mathrm{SNH}_{2}\right)$, 22.7 ( $2 \mathrm{x} \mathrm{CH}_{3}$ ), $20.3\left(1 \mathrm{x} \mathrm{CH}_{3}\right)$. FAB MS (3-nitrobenzyl alcohol): $m / z=208.0\left(\mathrm{C}_{8} \mathrm{H}_{18} \mathrm{SO}_{3} \mathrm{~N}\right.$ requires 208.3).

75 [2a][BPh ${ }_{4}$ ] Yield: 69\%. Found: C, 70.49; H, 7.01; N, 2.59. $\mathrm{C}_{32} \mathrm{H}_{38} \mathrm{O}_{3} \mathrm{SNB} .0 .25 \mathrm{CH}_{2} \mathrm{Cl}_{2}$ requires $\mathrm{C}, 70.59 ; \mathrm{H}, 7.03 ; \mathrm{N}$, $2.55 \%$. IR $v_{\max } / \mathrm{cm}^{-1}: 3284,3161,3059,2986,1580,1476$, 11323, 1112, 1065, 920, 846, 828, 756, 735, 702, 613. ${ }^{1} \mathrm{H}$ NMR (d - DMSO) $\delta_{\mathrm{H}}=7.18-6.80$ (br $m, 20 \mathrm{H}, \mathrm{BPh}_{4} \mathrm{Ar}-\mathrm{CH}$ ), 80 3.39-4.02 (m, 16H, CH$).{ }^{13} \mathrm{C}\left\{{ }^{1} \mathrm{H}\right\}$ NMR (d $\left.-\mathrm{DMSO}\right) \delta_{\mathrm{C}}=$ 163.6 (1:1:1:1 q, $\mathrm{BPh}_{4}$ ipso-C), 135.5, 125.3, $121.5(\mathrm{Ar}-\mathrm{C})$, 70.1, 69.7, $63.8\left(\mathrm{OCH}_{2}\right), 49.2\left(\mathrm{CH}_{2} \mathrm{SNH}_{2}\right)$. FAB MS (3nitrobenzyl alcohol): $m / z=208.0\left(\mathrm{C}_{8} \mathrm{H}_{18} \mathrm{O}_{3} \mathrm{SN}\right.$ requires 208.3).

85

\section{Reactions of [18]ane $\mathrm{O}_{4} \mathrm{~S}_{2}$ (3)}

[3a][mesSO I $_{3}$ ]: [18] aneO $_{4} \mathrm{~S}_{2}(0.750 \mathrm{~g}, 2.53 \mathrm{mmol})$ was dissolved in $\mathrm{CH}_{2} \mathrm{Cl}_{2} / \mathrm{MeOH}\left(1: 1,25 \mathrm{~cm}^{3}\right)$. Separately, $\mathrm{MSH}$ $90(0.545 \mathrm{~g}, 2.53 \mathrm{mmol})$ was dissolved in $\mathrm{CH}_{2} \mathrm{Cl}_{2}$ and added dropwise to the stirring thiacrown solution. After stirring overnight, the solution was reduced to $c a .2-3 \mathrm{~cm}^{3}$, after which $\mathrm{Et}_{2} \mathrm{O}\left(60 \mathrm{~cm}^{3}\right)$ was added and the resulting white solid filtered and dried in vacuo. Yield: $0.799 \mathrm{~g}$ (62\%). Found: C, 95 48.29; $\mathrm{H}, 6.81 ; \mathrm{N}, 3.45 ; \mathrm{C}_{21} \mathrm{H}_{37} \mathrm{O}_{7} \mathrm{~S}_{3} \mathrm{~N} \cdot 0.25 \mathrm{CH}_{2} \mathrm{Cl}_{2}$ requires $\mathrm{C}$, 47.89; H, 7.09; N, 2.63\%. IR $v_{\max } / \mathrm{cm}^{-1}$ : 3497, 3210 (br, -NH), 2936, 1473, 1456, 1209, 1175, 1088, 1016, 850 and 858, 682, 583, 549. ${ }^{1} \mathrm{H}$ NMR (d $\left.{ }^{6}-\mathrm{DMSO}\right): \delta_{\mathrm{H}}=6.78(\mathrm{~s}, 2 \mathrm{H}$, Ar C-H), $6.04\left(\mathrm{~s}, 2 \mathrm{H}, \mathrm{NH}_{2}\right), 3.37-4.00\left(\mathrm{~m}, 16 \mathrm{H}, \mathrm{CH}_{2}\right), 2.52(\mathrm{~s}, 6 \mathrm{H}, 2 \mathrm{x}$ $\left.100 \mathrm{Ar}-\mathrm{CH}_{3}\right), 2.18\left(\mathrm{~s}, 3 \mathrm{H}, \mathrm{Ar}-\mathrm{CH}_{3}\right) . \mathrm{ES}^{+} \mathrm{MS}(\mathrm{MeCN}): \mathrm{m} / \mathrm{z}=$ $312.5\left(\mathrm{C}_{12} \mathrm{H}_{26} \mathrm{~S}_{2} \mathrm{O}_{4} \mathrm{~N}\right.$ requires 312.5$)$.

[3a][BPh $4:: \quad\left\{[18]\right.$ aneO $\left._{4} \mathrm{~S}_{2}\left(\mathrm{NH}_{2}\right)_{2}\right\}\{\mathrm{MSH}\}_{2} \quad(0.529$ g, 0.73 mmol), was dissolved in $\mathrm{MeOH}\left(10 \mathrm{~cm}^{3}\right)$. Separately, $\mathrm{NaBPh}_{4}$ $105(0.250 \mathrm{~g}, 0.75 \mathrm{mmol})$ was dissolved in $\mathrm{MeOH}\left(5 \mathrm{~cm}^{3}\right)$ and added dropwise to the stirring sulfimide solution. A bright white solid appeared almost immediately, the mixture was allowed to stir for $c a .1 \mathrm{~h}$, after which the solid was filtered and dried in vacuo. Yield: $0.395 \mathrm{~g}$ (86\%). Found: C, 67.64; $\mathrm{H}$, 110 5.24; N, 1.82; $\mathrm{C}_{36} \mathrm{H}_{46} \mathrm{BNO}_{4} \mathrm{~S}_{2}$ requires $\mathrm{C}, 68.45 ; \mathrm{H}, 7.34 ; \mathrm{N}$, $2.22 \%$. IR $v_{\max } / \mathrm{cm}^{-1}: 3341,3221,3052,2983,1578,1477$, 1424, 1133, 1109, 1031, 845, 737, 709, 612. ${ }^{1} \mathrm{H}$ NMR $\left(\mathrm{d}^{6}-\right.$ DMSO): $\delta_{\mathrm{H}}=7.40-6.82$ (br $m, 20 \mathrm{H}, \mathrm{BPh}_{4} \mathrm{Ar}-\mathrm{CH}$ ), 3.43-4.05 $\left(m, 24 \mathrm{H}, \mathrm{CH}_{2}\right) . \mathrm{ES}^{+} \mathrm{MS}(\mathrm{MeCN}): \mathrm{m} / \mathrm{z}=312.5\left(\mathrm{C}_{12} \mathrm{H}_{26} \mathrm{~S}_{2} \mathrm{O}_{4} \mathrm{~N}\right.$ 115 requires 312.5$)$. 
[3b][mesSO $\left.]_{3}\right]_{2}$ was prepared using the same method as used for [4b][mesSO $\mathbf{H}_{3}$ ] except that 2.25 equivalents of $\mathrm{MSH}$ were employed. Yield: 0.834 g (71\%) Found: C, 48.36; H, 6.84; N, 5 3.78. $\mathrm{C}_{30} \mathrm{H}_{50} \mathrm{O}_{10} \mathrm{~S}_{4} \mathrm{~N}_{2} \cdot 0.25 \mathrm{CH}_{2} \mathrm{Cl}_{2}$ requires $\mathrm{C}, 48.56$; $\mathrm{H}, 6.80$; N, 3.74\%. IR $v_{\max } / \mathrm{cm}^{-1}$ : 3498, 3205, 2935, 1455, 1208, 1179, 1089, 1016, 858, 849, 682, 584, 549. ${ }^{1} \mathrm{H}$ NMR (d $\left.\mathrm{d}^{6}-\mathrm{DMSO}\right): \delta_{\mathrm{H}}$ $=6.77(\mathrm{~s}, 4 \mathrm{H}, \mathrm{Ar} \mathrm{C}-\mathrm{H}), 3.36-4.08\left(\mathrm{~m}, 24 \mathrm{H}, \mathrm{CH}_{2}\right), 2.51(\mathrm{~s}$, $\left.12 \mathrm{H}, \mathrm{CH}_{3}\right), 2.18\left(\mathrm{~s}, 6 \mathrm{H}, \mathrm{CH}_{3}\right) \cdot{ }^{13} \mathrm{C}\left\{{ }^{1} \mathrm{H}\right\}$ NMR (d $\left.-\mathrm{DMSO}\right): \delta_{\mathrm{C}}$ ${ }_{10}=142.4,136.5,135.9$ (quaternary $\mathrm{Ar}-\mathrm{C}$ ), $129.9(\mathrm{Ar} C-\mathrm{H})$, 69.1, 69.0, $62.1\left(\mathrm{OCH}_{2}\right), 47.5\left(\mathrm{SCH}_{2}\right), 22.7\left(2 \times \mathrm{CH}_{3}\right), 20.3$ (1 x $\left.\mathrm{CH}_{3}\right)$. FAB MS: $\mathrm{m} / \mathrm{z}=328.5\left(\mathrm{C}_{12} \mathrm{H}_{28} \mathrm{~S}_{2} \mathrm{O}_{4} \mathrm{~N}_{2}\right.$ requires 328.5).

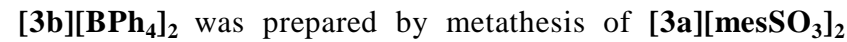
15 with $\mathrm{Na}\left[\mathrm{BPh}_{4}\right]$ in analogous manner to salts of 1a. Yield: 0.415 g, (90\%). Found: C, 73.11; H, 6.99; N, 3.01; $\mathrm{C}_{60} \mathrm{H}_{68} \mathrm{~B}_{2} \mathrm{~N}_{2} \mathrm{O}_{4} \mathrm{~S}_{2} . \mathrm{CH}_{3} \mathrm{OH}$ requires $\mathrm{C}, 73.34, \mathrm{H}, 7.26 ; \mathrm{N}$, 2.80\%. IR $v_{\max } / \mathrm{cm}^{-1}$ : 3340, 3220, 3053, 2915, 1578, 1477, 1424, 1132, 1424, 1133, 1098, 1031, 845, 737, 704, 612. ${ }^{1} \mathrm{H}$ 20 NMR (d - DMSO): $\delta_{\mathrm{H}}=7.40-6.82$ (br $m, 40 \mathrm{H}, \mathrm{Ar}-H$ ), 3.20-3.98 $\left(m, 24 \mathrm{H}, \mathrm{CH}_{2}\right) .{ }^{13} \mathrm{C}\left\{{ }^{1} \mathrm{H}\right\}$ NMR $\left(\mathrm{d}^{6}-\mathrm{DMSO}\right): \delta_{\mathrm{C}}=$ 164.1-162.6 (1:1:1:1 q, $\mathrm{BPh}_{4}$ ipso-C), 135.5, 125.3, 121.5 (Ar-C), 69.1, $62.1 \quad\left(\mathrm{OCH}_{2}\right), 47.6 \quad\left(\mathrm{H}_{2} \mathrm{NSCH}_{2}\right) . \mathrm{ES}^{+} \mathrm{MS}$ $(\mathrm{MeCN}): \mathrm{m} / \mathrm{z}=328.5\left(\mathrm{C}_{12} \mathrm{H}_{28} \mathrm{~S}_{2} \mathrm{O}_{4} \mathrm{~N}_{2}\right.$ requires 328.5$)$.

25

\section{Bridged systems}

$\left\{\left([9] \text { ane }{ }_{2} \mathbf{S}\right)_{2} \mathbf{N}\right\}\left[\mathrm{BPh}_{4}\right]$ 4: [1a][BPh $]$ (0.250 g, $\left.0.47 \mathrm{mmol}\right)$ was dissolved in THF $\left(15 \mathrm{~cm}^{3}\right)$, and the temperature reduced 30 to $-78^{\circ} \mathrm{C}$. LDA $\left(0.24 \mathrm{~cm}^{3}\right.$ of a $2 \mathrm{M}$ solution, $\left.0.47 \mathrm{mmol}\right)$ was added, and the mixture stirred for $1 \mathrm{~h}$. NBS (0.084 g, 0.47 mmol) was added as a solid, and after $1 \mathrm{~h}$ stirring, [9] ane $\mathrm{O}_{2} \mathrm{~S}$ ( $0.070 \mathrm{~g}, 0.47 \mathrm{mmol})$ was added. The yellow solution was stirred overnight, during which time the temperature was 35 gradually raised to r.t. The solution was reduced to dryness in vacuo, after which $\mathrm{H}_{2} \mathrm{O}\left(25 \mathrm{~cm}^{3}\right)$ was added along with $\mathrm{CH}_{2} \mathrm{Cl}_{2}\left(2 \times 25 \mathrm{~cm}^{3}\right)$. The combined organic layers were dried over $\mathrm{MgSO}_{4}$, reduced to ca. 2-3 $\mathrm{cm}^{3}$, and $\mathrm{Et}_{2} \mathrm{O}\left(60 \mathrm{~cm}^{3}\right)$ added. The resulting off-white solid was filtered and dried in 40 vacuo. Yield: 0.127 g (43\%). Found: C, 69.45; H, 7.30; N, 1.84. $\mathrm{C}_{36} \mathrm{H}_{44} \mathrm{BNO}_{4} \mathrm{~S}_{2}$ requires $\mathrm{C}, 68.67 ; \mathrm{H}, 7.04 ; \mathrm{N}, 2.22 \%$. IR $v_{\max } / \mathrm{cm}^{-1}:$ 3422, 1683, 1478, 1426, 1391, 1300, 1203, 1153, 1087, 940, 927, 858, 736, 707. ${ }^{1} \mathrm{H}$ NMR (d $\left.-\mathrm{DMSO}\right): \delta_{\mathrm{H}}=$ 7.21-6.76 (br $\left.m, 20 \mathrm{H}, \mathrm{BPh}_{4} \mathrm{Ar}-\mathrm{CH}\right), 3.17\left(m, 24 \mathrm{H}, \mathrm{CH}_{2}\right.$ ). ${ }_{45}{ }^{13} \mathrm{C}\left\{{ }^{1} \mathrm{H}\right\}$ NMR (d $\left.-\mathrm{DMSO}\right): \delta_{\mathrm{C}}=163.6$ (1:1:1:1 q, BPh $\left.\mathrm{Br}-\mathrm{C}\right)$, 135.4, 124.9, $121.2(\mathrm{Ar}-\mathrm{C}), 69.7,63.8\left(\mathrm{OCH}_{2}\right), 48.2$ $\left(\mathrm{NSCH}_{2}\right)$. FAB MS (3-nitrobenzyl alcohol): $\mathrm{m} / \mathrm{z}=310.0$ $\left(\mathrm{C}_{12} \mathrm{H}_{24} \mathrm{O}_{4} \mathrm{~S}_{2} \mathrm{~N}\right.$ requires 310.4$)$.

${ }_{50}\left\{\left([\mathbf{1 2}] \text { ane } \mathbf{O}_{3} \mathbf{S}\right)_{2} \mathbf{N}\right\}\left[\mathbf{B P h}_{\mathbf{4}}\right] \mathbf{5}$ was prepared using the same method as for 4, using [2a] $\left[\mathbf{B P h}_{\mathbf{4}}\right]$. Yield: $0.101 \mathrm{~g}$ (38\%). Found: C, 66.38; H, 7.06; N, 2.49. $\mathrm{C}_{40} \mathrm{H}_{52} \mathrm{O}_{6} \mathrm{~S}_{2} \mathrm{NB}$ requires $\mathrm{C}$, 66.93; H, 7.30; N, 1.95\%; IR $v_{\max } / \mathrm{cm}^{-1}: 3284,3161,3055$, 3035, 2926, 2871, 1578, 1475, 1457, 1423, 1397, 1154, 1136, 55 1107, 1092, 927, 844, 733, 710, 612. ${ }^{1} \mathrm{H}$ NMR (d $\left.{ }^{6}-\mathrm{DMSO}\right): \delta_{\mathrm{H}}$ = 7.19-6.81 (br m, 20H, $\left.\mathrm{BPh}_{4} \mathrm{Ar}-\mathrm{CH}\right), 3.24-4.02(\mathrm{~m}, 12 \mathrm{H}$, $\left.\mathrm{CH}_{2}\right) .{ }^{13} \mathrm{C}\left\{{ }^{1} \mathrm{H}\right\}$ NMR $\left(\mathrm{d}^{6}-\mathrm{DMSO}\right): \delta_{\mathrm{C}}=163.6\left(1: 1: 1: 1 q, \mathrm{BPh}_{4}\right.$ ipso-C), 135.5, 125.3, 121.5 ( $\mathrm{Ar}-\mathrm{C}), 69.9,69.6,64.3\left(\mathrm{OCH}_{2}\right)$,
$51.9\left(\mathrm{NSCH}_{2}\right) . \mathrm{ES}^{+} \mathrm{MS}(\mathrm{MeCN}): \mathrm{m} / \mathrm{z}=398.2\left(\mathrm{C}_{16} \mathrm{H}_{32} \mathrm{~S}_{2} \mathrm{O}_{6} \mathrm{~N}\right.$ 60 requires 398.6); $\mathrm{ES}^{-} \mathrm{MS}: \mathrm{m} / \mathrm{z}=319.2\left(\mathrm{C}_{24} \mathrm{H}_{20} \mathrm{~B}\right.$ requires 319.2).

\{([18]aneO $\left.\left.\mathbf{S}_{4}\right) \mathbf{N}\right\}\left[\mathrm{BPh}_{4}\right]$ 6: [3a][BPh $\left.{ }_{4}\right](0.250 \mathrm{~g}, 0.26 \mathrm{mmol})$ 65 was dissolved in THF $\left(15 \mathrm{~cm}^{3}\right)$, the temperature reduced to $-78^{\circ} \mathrm{C}$ and LDA $\left(0.13 \mathrm{~cm}^{3}\right.$ of a $2 \mathrm{M}$ solution, $\left.0.26 \mathrm{mmol}\right)$ was added. After stirring for $30 \mathrm{~min} .$, NBS (0.046 g, $0.26 \mathrm{mmol}$ ) was added as a solid, and the mixture stirred overnight, slowly warming to room temperature. The mixture was reduced to 70 dryness under reduced pressure, $\mathrm{H}_{2} \mathrm{O}\left(25 \mathrm{~cm}^{3}\right)$ added, and the product extracted into $\mathrm{CH}_{2} \mathrm{Cl}_{2}\left(2 \mathrm{x} 25 \mathrm{~cm}^{3}\right)$. The combined organic extracts were dried over $\mathrm{MgSO}_{4}$, reduced to ca. 2-3 $\mathrm{cm}^{3}$, and $\mathrm{Et}_{2} \mathrm{O}$ added. The resulting off-white precipitate was filtered and dried in vacuo. Yield: $0.098 \mathrm{~g}$ (60\%). Found: C, ${ }_{75} 67.41 ; \mathrm{H}, 6.71 ; \mathrm{N}, 3.15 ; \mathrm{C}_{36} \mathrm{H}_{44} \mathrm{BNO}_{4} \mathrm{~S}_{2} \cdot 0.25 \mathrm{CH}_{2} \mathrm{Cl}_{2}$ requires C, 66.89; H, 6.89; N, 2.15\%. IR $v_{\max } / \mathrm{cm}^{-1}: 2853,1457,1376$, 1210, 1179, 1087, 1014, 918, 858 681. ${ }^{1} \mathrm{H}$ NMR (d ${ }^{6}$-DMSO): $\delta_{\mathrm{H}}=7.38-6.79$ (br $m, 20 \mathrm{H}, \mathrm{BPh}_{4} \mathrm{Ar}-\mathrm{CH}$ ), 3.30-3.50 (br $m$, $\left.24 \mathrm{H}, \mathrm{CH}_{2}\right) \cdot{ }^{13} \mathrm{C}\left\{{ }^{1} \mathrm{H}\right\}$ NMR $\left(\mathrm{d}^{6}-\mathrm{DMSO}\right): \delta_{\mathrm{C}}=163.6(1: 1: 1: 1 q$, $80 \mathrm{BPh}_{4}$ ipso-C)), 135.5, 125.3, 121.5 (Ar-C), 68.7, 64.3 $\left(\mathrm{OCH}_{2}\right), 51.1\left(\mathrm{NSCH}_{2}\right) \cdot \mathrm{FAB}^{+} \mathrm{MS}$ (3-nitrobenzyl alcohol): $\mathrm{m} / \mathrm{z}=310.5\left(\mathrm{C}_{12} \mathrm{H}_{24} \mathrm{~S}_{2} \mathrm{O}_{4} \mathrm{~N}\right.$ requires 310.5).

\section{X-Ray Crystallography}

85 Details of the crystallographic data collection and refinement parameters are given in Table 1 . Data for [2a][BPh $\left.\mathbf{B}_{4}\right]$ and [6][BPh $\mathbf{B P}_{\mathbf{4}}$ ] were collected using a Bruker AXS SMART 1000 CCD area-detector diffractometer using sealed-tube graphitemonochromated $\mathrm{Mo}-\mathrm{K}_{\alpha}$ radiation and narrow frame 90 exposures $\left(0.3^{\circ}\right)$. Cell parameters were refined from the observed $(\omega)$ angles of all strong reflections in each data set. Intensities were corrected semi-empirically for absorption, based on symmetry-equivalent and repeated reflections. All structures were solved by direct methods and refined on $F^{2}$ 95 values for all unique data by full-matrix least-squares. ${ }^{9}$ Data for $[\mathbf{3 b}]\left[\mathbf{B P h}_{\mathbf{4}}\right],[\mathbf{4}]\left[\mathbf{B P h}_{\mathbf{4}}\right]$ and $[\mathbf{5}]\left[\mathbf{B P h}_{\mathbf{4}}\right]$ were collected at 93 $\mathrm{K}$ by using a Rigaku MM007 High brilliance RA generator (Mo K $\alpha$ radiation, confocal optics) and Mercury CCD system. At least a full hemisphere of data was collected using $\omega$ or $100 \omega / \varphi$ scans. Intensities were corrected for Lorentz-polarisation and for absorption. The structures were solved by direct methods. Hydrogen atoms bound to carbon were idealised. Structural refinements were performed with full-matrix leastsquares based on $\mathrm{F}^{2}$ by using the program SHELXTL. ${ }^{\mathbf{1 0 , 1 1}}$

105

\section{Supplementary data}

Crystallographic data have been deposited with the Cambridge Crystallographic Data Centre as supplementary publication numbers CCDC XXX XXXX. Copies of the data 110 can be obtained free of charge on application to CCDC, 12 Union Road, Cambridge CB2 1EZ, UK (Fax: +44-1223336033; email: deposit@ccdc.cam.ac.uk or www: http://www.ccdc.cam.ac.uk). 


\section{Results and discussion}

Reaction of crowns $\mathbf{1}$ and 2 with $\mathrm{MSH}$ in $\mathrm{Et}_{2} \mathrm{O}$ results in rapid 5 formation of the $\left[\mathrm{mesSO}_{3}\right]^{-}$salts of the aminated cations $\left\{[9] \mathrm{aneO}_{2} \mathrm{~S}\left(\mathrm{NH}_{2}\right)\right\}^{+} \mathbf{1 a}$ and $\left\{[12] \mathrm{aneO}_{3} \mathrm{~S}\left(\mathrm{NH}_{2}\right)\right\}^{+}$2a, both of which precipitate from the ether as waxy, colourless solids (Scheme 2). In both cases, treatment of a methanolic solution of the material with a slight excess of $\mathrm{Na}\left[\mathrm{BPh}_{4}\right]$ readily 10 precipitates the corresponding $\left[\mathrm{BPh}_{4}\right]^{-}$salts; these are more crystalline in nature and more readily handled. The sulfimidation and metathesis reactions proceed very cleanly, giving yields often in excess of $80 \%$. Such results echo those we reported previous for the analogous reaction of the larger ${ }_{15}$ crown [18]ane ${ }_{5} \mathrm{~S},{ }^{11}$ thereby confirming that the amination reactions are equally viable for all ring sizes.

Slow diffusion of $\mathrm{Et}_{2} \mathrm{O}$ into a $\mathrm{CDCl}_{3} / \mathrm{dmso}$ solution of [2a] $\left[\mathrm{BPh}_{4}\right]$ generated crystals suitable for X-ray crystallography, and the latter technique confirms the 20 expected structure (Fig.2). The $\mathrm{S}-\mathrm{N}$ bond length in this case (1.635(14) $\AA$ ) is in the range expected for sulfimidium units. The sulfimidium $\mathrm{NH}_{2}{ }^{+}$unit is oriented exo to the ring, with no intra- or intermolecular $\mathrm{H}$-bonding interactions to the ether $\mathrm{O}$ atoms evident.

25 Four resonances are seen in the solution ${ }^{13} \mathrm{C}\left\{{ }^{1} \mathrm{H}\right\}$ NMR spectrum as expected (indicating the time-averaged symmetry in solution). Also as expected, within the spectrum of [2a] $\left[\mathrm{BPh}_{4}\right]$ resonances associated with the $\mathrm{SCH}_{2}$ carbon atom are most affected by the sulfimidation reaction, shifting 30 substantially (by ca. $15 \mathrm{ppm}$ ) to high frequency - undoubtedly a consequence of the presence of formally S(IV) in the product (cf. S(II) in the parent crown).

The ease with which colourless, powdered $\left[\mathrm{BPh}_{4}\right]^{-}$salts of 1a and 2a can be obtained by metathesis, is a key to further 35 reactivity. Not only are such compounds easier to handle than the corresponding $\left[\mathrm{mesSO}_{3}\right]^{-}$salts, they also possess inert counterions; in our experience the $\left[\mathrm{mesSO}_{3}\right]^{-}$is often less than "innocent" in terms of reactivity. Treatment of a solution of [1a] $\left[\mathrm{BPh}_{4}\right]$ or $[\mathbf{2 a}]\left[\mathrm{BPh}_{4}\right]$ with LDA in diethyl ether solution 40 at $-78^{\circ} \mathrm{C}$, followed by bromination using NBS and addition of a further equivalent of the parent thia/oxa crown leads to (Scheme 3) the formation of the intermolecularly N-bridged bicylic cations $\left\{\left([9] \text { aneO }_{2} \mathrm{~S}\right)_{2} \mathrm{~N}\right\}^{+}(4)$ and $\left\{\left([12] \mathrm{aneO}_{3} \mathrm{~S}\right)_{2} \mathrm{~N}\right\}^{+}$ (5), which are readily isolated as their $\left[\mathrm{BPh}_{4}\right]^{-}$salts. These ${ }_{45}$ compounds have also been characterised by ${ }^{1} \mathrm{H}$ and ${ }^{13} \mathrm{C}\left\{{ }^{1} \mathrm{H}\right\}$ NMR and IR spectroscopy, mass spectrometry and microanalyses. We note that formation of the $\mathrm{S}-\mathrm{N}-\mathrm{S}$ bridge leads to a further small high frequency shift for the $\mathrm{SCH}_{2}$ group in the ${ }^{13} \mathrm{C}\left\{{ }^{1} \mathrm{H}\right\}$ NMR spectrum compared to the 50 corresponding sulfimidium cations. The crystal structures of the $\left[\mathrm{BPh}_{4}\right]^{-}$salts of 4 (Fig. 3) and 5 (Fig 4) provide further evidence for the formulations of these unusual examples of coupled macrocyclic species (albeit the crystal quality of the latter is rather poor, hence detailed comparisons of bond 55 lengths etc are not justified, though connectivity is certainly established). As noted earlier, the ${ }^{13} \mathrm{C}\left\{{ }^{1} \mathrm{H}\right\}$ spectra show a number of lines equivalent to the number of chemically independent carbons. Thus while the solid state structure in Fig. 3 is clearly asymmetric in terms of the disposition of the 60 carbons about the central nitrogen, in solution the environments are equivalent, hence only three ${ }^{13} \mathrm{C}$ signals are observed. The two pairs of crystallographically independent $\mathrm{S}-\mathrm{N}$ bond distances within cation $\mathbf{4}$ and cation $\mathbf{5}$ are very similar (ca. $1.64 \AA$ ), consistent with delocalisation of electron 65 density across the SNS linkage. These distances are ca. $0.02 \AA$ longer than $\mathrm{d}(\mathrm{S}-\mathrm{N})$ within the sulfimidium cation, $\left\{[12] \text { aneO }_{3} \mathrm{~S}\left(\mathrm{NH}_{2}\right)\right\}^{+}$.

In contrast to the larger system $\left\{\left([18] \operatorname{aneO}_{5} \mathrm{~S}\right)_{2} \mathrm{~N}\right\}^{+},{ }^{\mathbf{1 1}}$ we have not seen any evidence for coordination of $\mathrm{Na}^{+}$upon 70 addition of excess $\mathrm{Na}\left[\mathrm{BPh}_{4}\right]$ to either $\mathbf{4}$ or $\mathbf{5}$. Thus crystallisation of $1: 1$ mixtures of $\mathbf{4}$ or 5 with $\mathrm{Na}\left[\mathrm{BPh}_{4}\right]$ in methanol, by slow ether diffusion, simply yields crystals of the starting materials. This contrast in results presumably reflects the fact that smaller rings are involved in these 75 systems. In the sodium adduct of $\left\{\left([18] \mathrm{aneO}_{5} \mathrm{~S}\right) \mathrm{N}\right\}^{+}$all five oxygen atoms of one ring exhibit significant interactions with the sodium cation, but only two (the most distant from the sulfur) from the other ring are involved in bonding. So even with such large rings present, the limiting action of the 80 "hinge" at the nitrogen atom precludes symmetric coordination to the enclosed sodium. It is therefore not surprising that within $\mathbf{4}$ and $\mathbf{5}$ encapsulation of the sodium is not observed.

In the case of the dithiacrown 3 the presence of two 85 potential reaction sites provides the opportunity for variation on the sulfimidation reactions seen thus far. The simplest reaction is, of course, that with one molar equivalent of $\mathrm{MSH}$, and as expected this results in amination of one of the sulfur atoms to give the ionic species $\left\{[18] \mathrm{aneO}_{4} \mathrm{~S}_{2}\left(\mathrm{NH}_{2}\right)\right\}^{+}$(3a) in 90 good yield (Scheme 2). As with the previous examples, addition of a slight excess of $\mathrm{Na}\left[\mathrm{BPh}_{4}\right]$ to solutions of the $\left[\mathrm{mesSO}_{3}\right]^{-}$salt results in the formation of $[3 \mathbf{3 a}]\left[\mathrm{BPh}_{4}\right]$. The latter was characterised by ${ }^{1} \mathrm{H}$ NMR and IR spectroscopy, $\mathrm{ES}^{+}$ MS and by microanalysis.

95 The reaction of MSH with $\mathbf{3}$ also generates small amounts of the dication which results from oxidation of both ring sulfurs by $\mathrm{MSH}$ (as shown by mass spectrometry). This product may be prepared directly by reaction of [18]aneO $\mathrm{S}_{2}$ with two equivalents of $\mathrm{MSH}$, thereby generating $100\left\{[18] a_{a n e O}\left(\mathrm{~S}=\mathrm{NH}_{2}\right)_{2}\right\}^{2+}(3 \mathbf{b})$ cation in excellent yield. This observation indicates that although cation 3a has a positive charge this does not preclude further reaction and increase in this charge. The ${ }^{13} \mathrm{C}\left\{{ }^{1} \mathrm{H}\right\}$ NMR spectrum of $\mathbf{3 b}$ reflects the high solution symmetry of the cation, leading to three distinct 105 resonances; two due to the $\mathrm{CH}_{2} \mathrm{O}$ groups at 69.1 and $62.1 \mathrm{ppm}$ and the third at $47.6 \mathrm{ppm}$ due to the $\mathrm{H}_{2} \mathrm{NSCH}_{2}$ unit. Further authentication of this species comes from its $\mathrm{X}$-ray structure which is shown in Fig. 5. The $\left\{[18] \mathrm{aneO}_{4}\left(\mathrm{~S}=\mathrm{NH}_{2}\right)_{2}\right\}^{2+}$ cation is centrosymmetric, with the $\mathrm{NH}_{2}{ }^{+}$units oriented in opposite 110 directions relative to the $\mathrm{O}_{4} \mathrm{~S}_{2}$ plane. The $\mathrm{S}-\mathrm{N}$ bond distance (1.624(4) $\AA$ ) is similar to that in 2a and related protonated sulfimidium species within this work.

The sulfimidium cation 3a can be deprotonated by addition of LDA at low temperatures to give the 'free' sulfimide in 115 situ, which can subsequently be brominated using NBS. Upon 
bromination, the presence of the second thioether $\mathrm{S}$ atom enables the pendant $\mathrm{S}=\mathrm{NBr}$ group to bridge across the molecule to form the $\left\{\left([18] \mathrm{aneO}_{4} \mathrm{~S}_{2}\right) \mu-\mathrm{N}\right\}^{+}$cation (6) via an intramolecular process. The spectroscopic features of this 5 product parallel those for the other species in this work, with the $\mathrm{ES}^{+} \mathrm{MS}$ clearly showing the cation peak two mass units below that for the sulfimidium cation $\left\{[18] \text { ane } \mathrm{O}_{4} \mathrm{~S}_{2}\left(\mathrm{NH}_{2}\right)\right\}^{+}$as expected. Crystals of $[6]\left[\mathrm{BPh}_{4}\right]$ suitable for $\mathrm{X}$-ray crystallography were grown by vapour diffusion of $\mathrm{Et}_{2} \mathrm{O}$ into $10 \mathrm{a} \mathrm{CDCl}_{3} / \mathrm{dmso}$ solution. As Fig. 6 shows, the molecule forms a 'basket' shape, with the $\mathrm{S}-\mathrm{N}-\mathrm{S}$ linkage bridged over the molecule. The nitrogen atom lies, on average $2.96 \AA$ above the plane of the four oxygen atoms. The S-N bond lengths are very similar to each other [1.6465(17) and 1.6469(17) $\AA$ ], and 15 to those of other compounds containing 'bridged' sulfimide groups (vide supra). As Fig.6 highlights, the structure of the cation effectively consists of two, fused, 11-membered macrocycles, and it is worth noting that the individual macrocyles are themselves unique. The formation of this 20 cation thus mirrors the formation of $\left\{[9] \operatorname{aneS}\left(\mathrm{NH}_{2}\right) \mathrm{S}_{2}(\mu-\mathrm{N})\right\}^{2+}$ in the reaction of [9]ane $\mathrm{S}_{3}$ with excess $\mathrm{MSH}^{12}$ In the latter case the formation of the transannular bridge was unexpected; it most likely occurs via loss of $\left[\mathrm{NH}_{4}\right]^{+}$from the initial product, $\left\{[9] \text { ane }\left(\mathrm{SNH}_{2}\right)_{3}\right\}^{3+}$. In contrast, the formation of (6) 25 is a rational synthesis that can be expected to be applicable to all crown systems bearing two sulfur atoms.

\section{Conclusions}

The formation of the simple sulfimidium derivatives of 30 crowns 1-3 confirms the general viability of $\mathrm{MSH}$ as aminating agent for mixed thia/oxa crown systems. In conjunction with the preliminary work on reactions of [18] $\mathrm{aneO}_{5} \mathrm{~S}$ it confirms that this constitutes a very effective new route for derivatisation of such crowns. The amenable 35 bridging reactions have great potential for further utilisation, both from the point of the view of the linked crown systems such as $\mathbf{4}$ and the intramolecularly bridged species such as $\mathbf{6}$. In terms of the former class of compound, it is clear that a wide variety of arrangements is possible, depending upon the 40 crowns chosen as starting materials. The demonstrated ability of the larger ring systems to coordinate to a $\mathrm{Na}^{+}$guest ion indicates that a significant new area of macrocyclic coordination chemistry should result from this approach. In terms of the intramolecular bridge motif it is clear that many

45 variations on 6 should be possible. The presence of unique macrocycles within this structure also suggests that linking of the ends of chain thia/oxa ethers should be possible, with sulfimide nitrogen atoms forming bridges that generate new cyclic structures. Work on all these fascinating possibilities is 50 underway.

\section{Acknowledgements}

This work was supported by the EPSRC (EP/C510100); we acknowledge the use of the EPSRC Mass Spectrometry Service at 55 the University of Wales, Swansea.

\section{Notes and references}

${ }^{a}$ Department of Chemistry, Loughborough University, Leics. LE11 3TU, UK.E-mail: P.F.Kelly@lboro.ac.uk

${ }_{60}{ }^{b}$ School of Chemistry, University of Southampton, Southampton, SO17 $1 B J, U K$.

${ }^{c}$ School of Chemistry, University of St. Andrews, St. Andrews, Fife, KY16 9ST, UK

$65 \dagger$ Electronic Supplementary Information (ESI) available: [details of any supplementary information available should be included here]. See DOI: $10.1039 / \mathrm{b} 000000 \mathrm{x} /$

1 L. F. Lindoy, in The Chemistry of Macrocycle Ligand Compounds, 70 Cambridge University Press, Cambridge, 1989; R. D. Rogers and C. B. Bauer, in Comprehensive Supramolecular Chemistry, J. L. Atwood, J. E. D. Davies, D. D. MacNicol and F. Vögtle (eds), 1996, 1, 315;T. P. Hanusa, in Comprehensive Coordination Chemistry II, J. A. McCleverty, T. J. Meyer (eds), Elsevier, Amsterdam, 2004, 3, 1.

752 a) M. J. Hesford, W. Levason, M. L. Matthews and G. Reid, J. Chem. Soc., Dalton Trans., 2003, 2852; b) C. D. Beard, L. Carr, M. F. Davis, J. Evans, W. Levason, L. D. Norman, G. Reid and M. Webster, Eur. J. Inorg. Chem., 2006, 4399; c) S. C. Davies, M. C. Durrant, D. L. Hughes, C. LeFloc'h, S. J. A. Pope, G. Reid, R. L.

80 Richards and J. R. Sanders, J. Chem. Soc., Dalton Trans., 1998, 2191.

3 J. R. Dann, P. P. Chiesa and J. W. Gates, J. Org. Chem., 1961, 26, 1991.

4 a) E. V. Fesenko, L. I. Budarin, E. S. Levchenko, Yu. A. Serguchev 85 and A. M. Neduv, Koordinatsionnaya Khimiya, 1986, 12, 891; b) S. V. Pavlova and S. I. Tyukhtenko, Teoreticheskaya $i$ Eksperimental'naya Khimiya, 1991, 27, 170; c) T. I. Petrenko, S. N. Gaidamaka and Yu. A. Serguchev, Teoreticheskaya i Eksperimental'naya Khimiya, 1988, 24, 191.

905 a) H.-J. Küppers, K. Wieghardt, B. Nuber, J. Weiss, E. Bill and A. X. Trautwein, Inorg. Chem., 1987, 26, 3762; b) A. J. Blake, M. Schrőder and M. J. Sullivan, Acta Crystallogr., Sect. C, 1991, 47, 2717.

6 M. R. J. Elsegood, P. F. Kelly, G. Reid and P. M.Staniland, Dalton Trans., 2007, 1665.

957 J. D. Lamb, R. M. Izatt, C. S. Swain and J. J. Christensen, J. Am. Chem. Soc., 1980, 102, 475.

8 Y.Tamura, J.Minamikawa, K.Sumoto, S.Fujii and M.Ikeda, J.Org.Chem. 1973, 38, 1239.

9 103. G. M. Sheldrick, SHELXTL user manual, version 6.10, Bruker ACS Inc., Madison, WI, 2000.

10 SHELXTL 6.14 G M Sheldrick, Bruker AXS Madison 2004.

11. G. M. Sheldrick, Acta Crystallogr., 2008, A64, 112-122.

12 M.R.J Elsegood, K.E.Holmes, L.M.Gilby \& P.F.Kelly, Can..J.Chem. 2002, 18, 1410.
110

115 


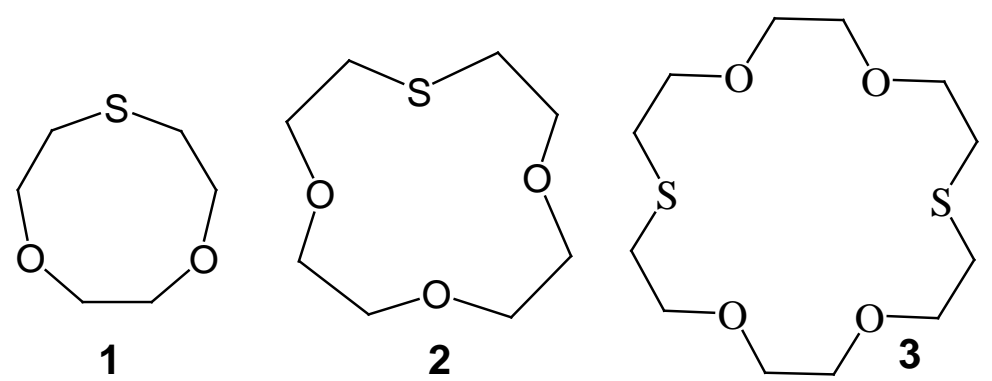

35

40

Fig.1 The structure of crowns 1-3.

50 

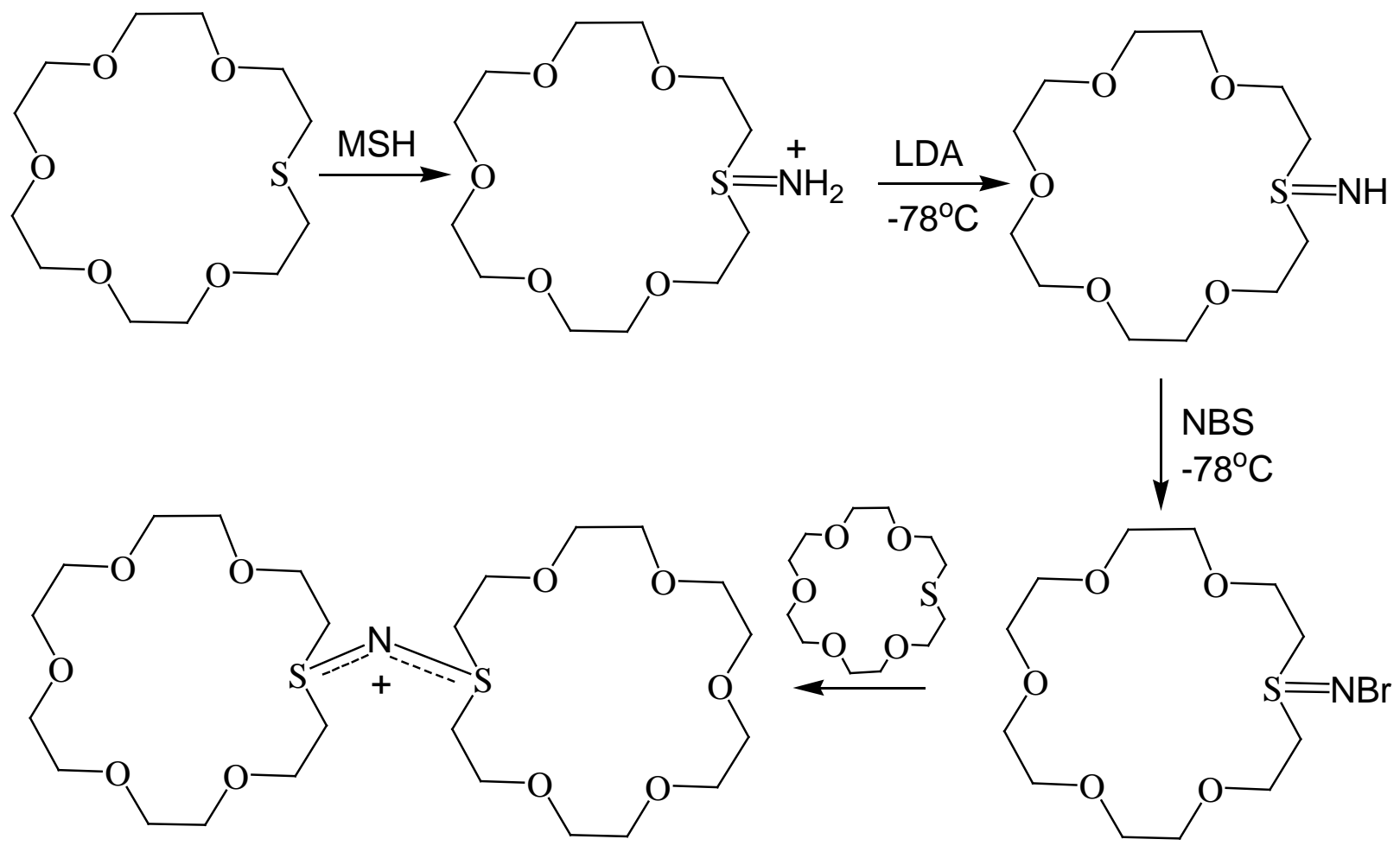

Scheme 1 Linkage reactions of [18] $\mathrm{aneO}_{5} \mathrm{~S}$ performed in our initial study. ${ }^{11}$

10 


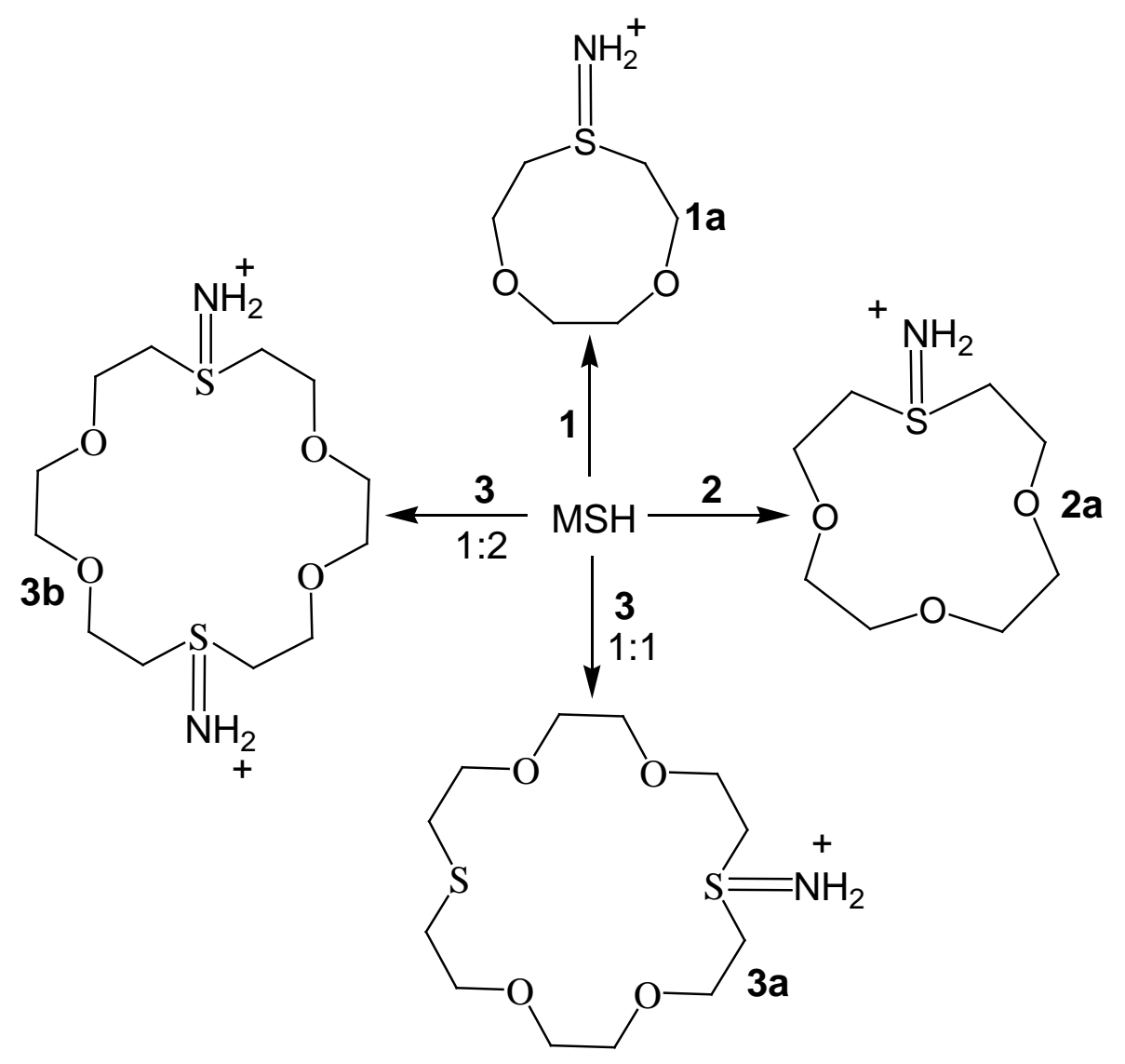

Scheme 2 Sulfimidation reactions of 1-3 using MSH. 


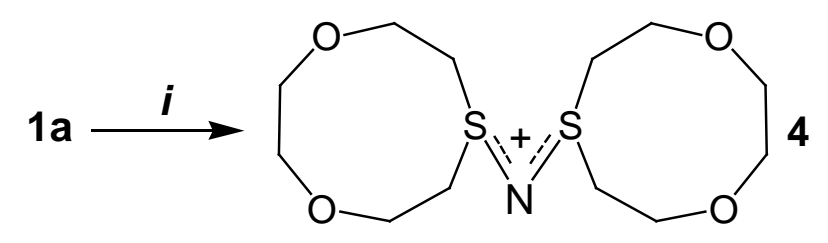

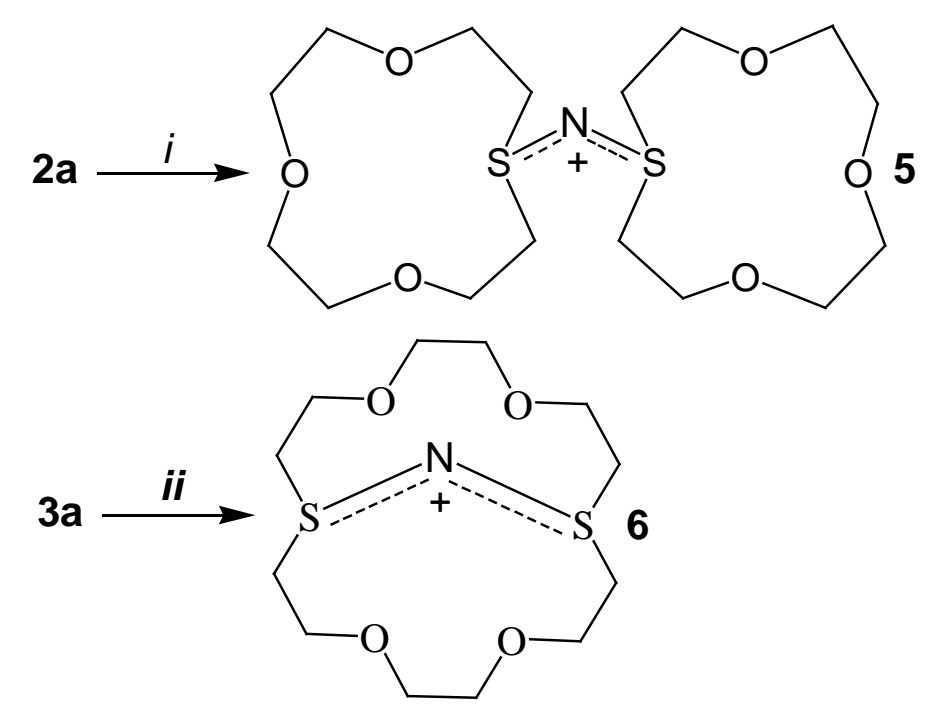

5 Scheme 3 Bridging reactions of crown sulfimidium salts. $\boldsymbol{i}$ LDA, NBS $\left(-78^{\circ} \mathrm{C}\right)$ followed by addition of parent crown; $\boldsymbol{i}$ LDA, NBS $\left(-78^{\circ} \mathrm{C}\right)$.

10 


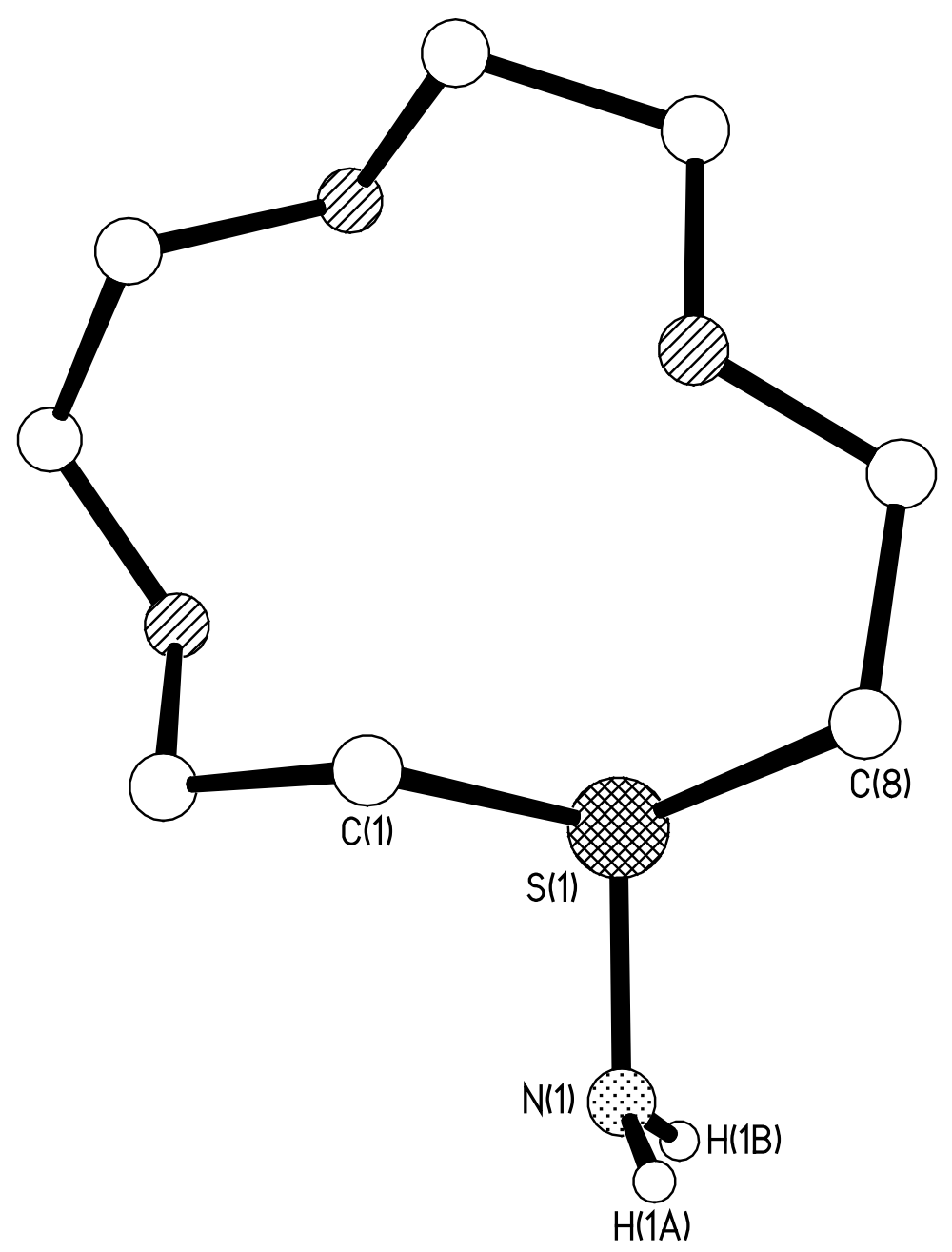

${ }_{5}$ Fig.2 View of the structure of the cation within [2a][ $\left.\mathrm{BPh}_{4}\right]$ (in all figures crossed lined atoms are oxygen; most $\mathrm{H}$ atoms and any disorder are omitted for clarity). 


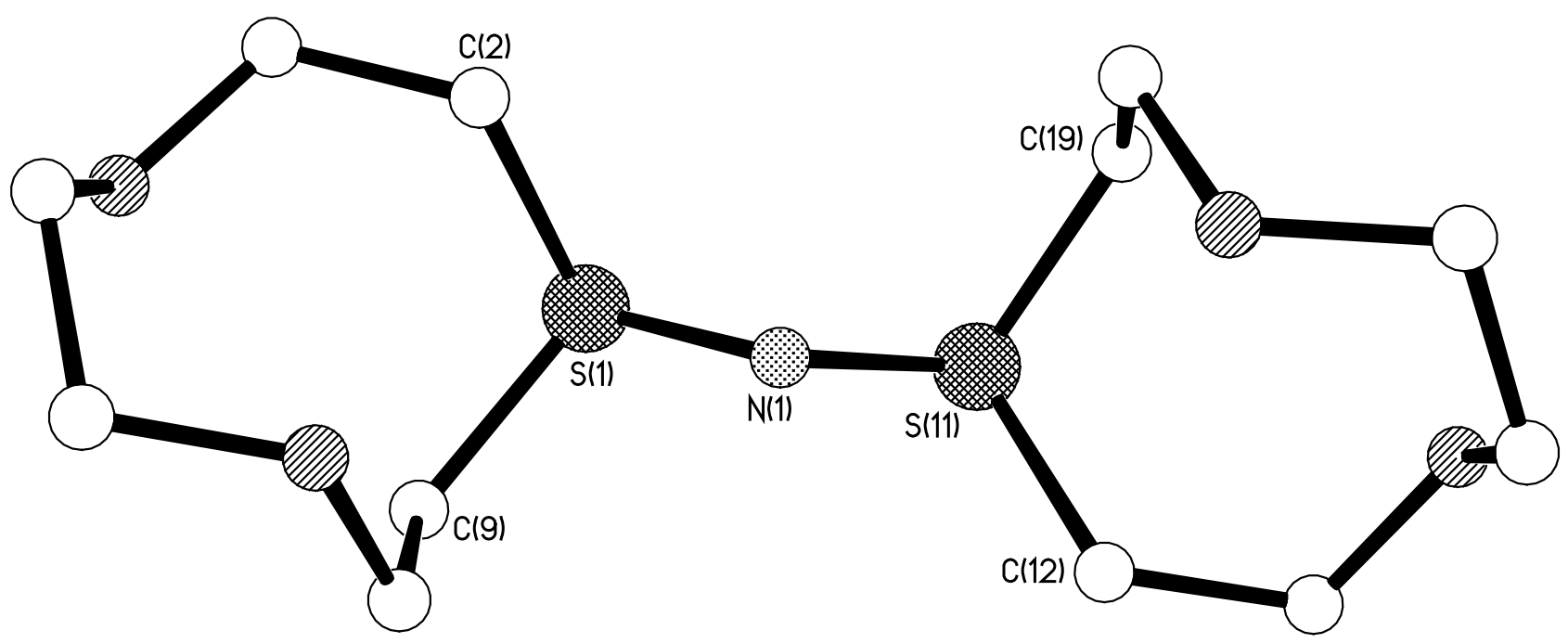

Fig.3 View of the structure of the cation in $[4]\left[\mathrm{BPh}_{4}\right]$. 


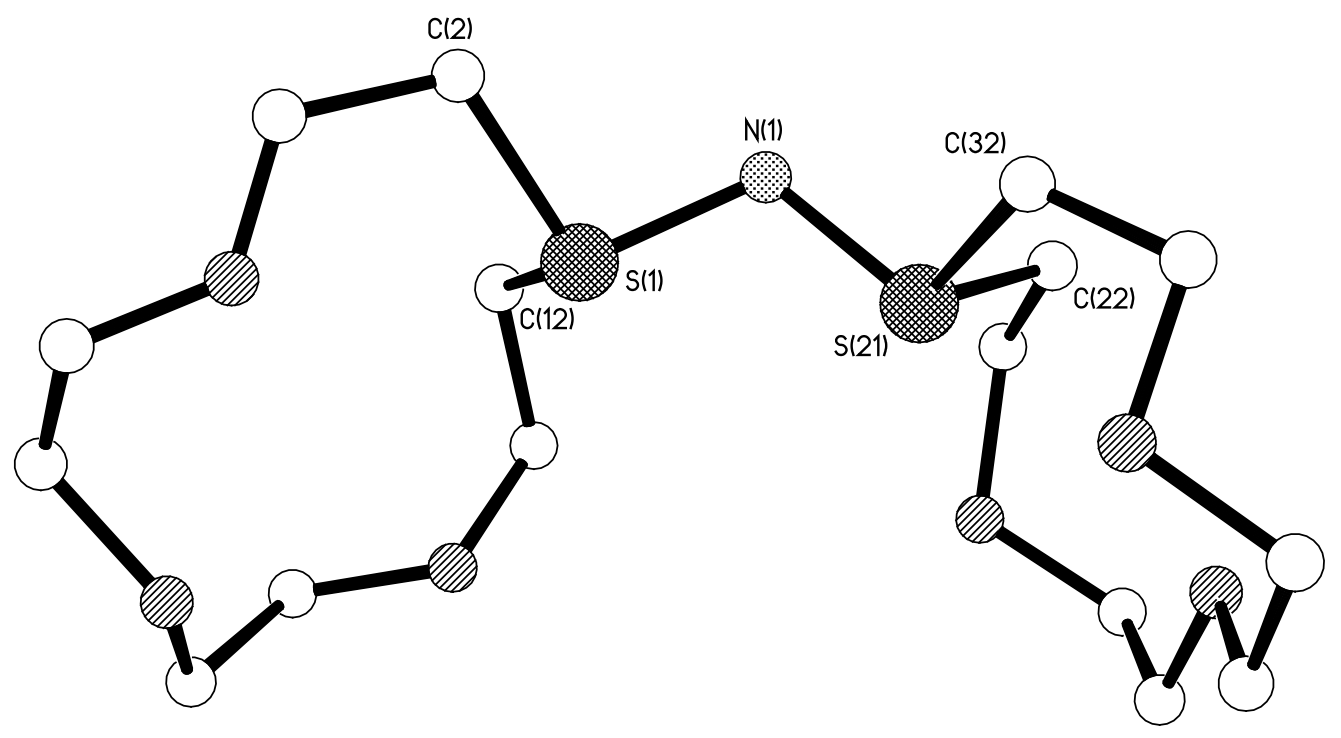

10

Fig. $4 \mathrm{X}$-ray crystal structure of the cation within $[5]\left[\mathrm{BPh}_{4}\right] \cdot \mathrm{CHCl}_{3}$ with atom numbering scheme.

15 


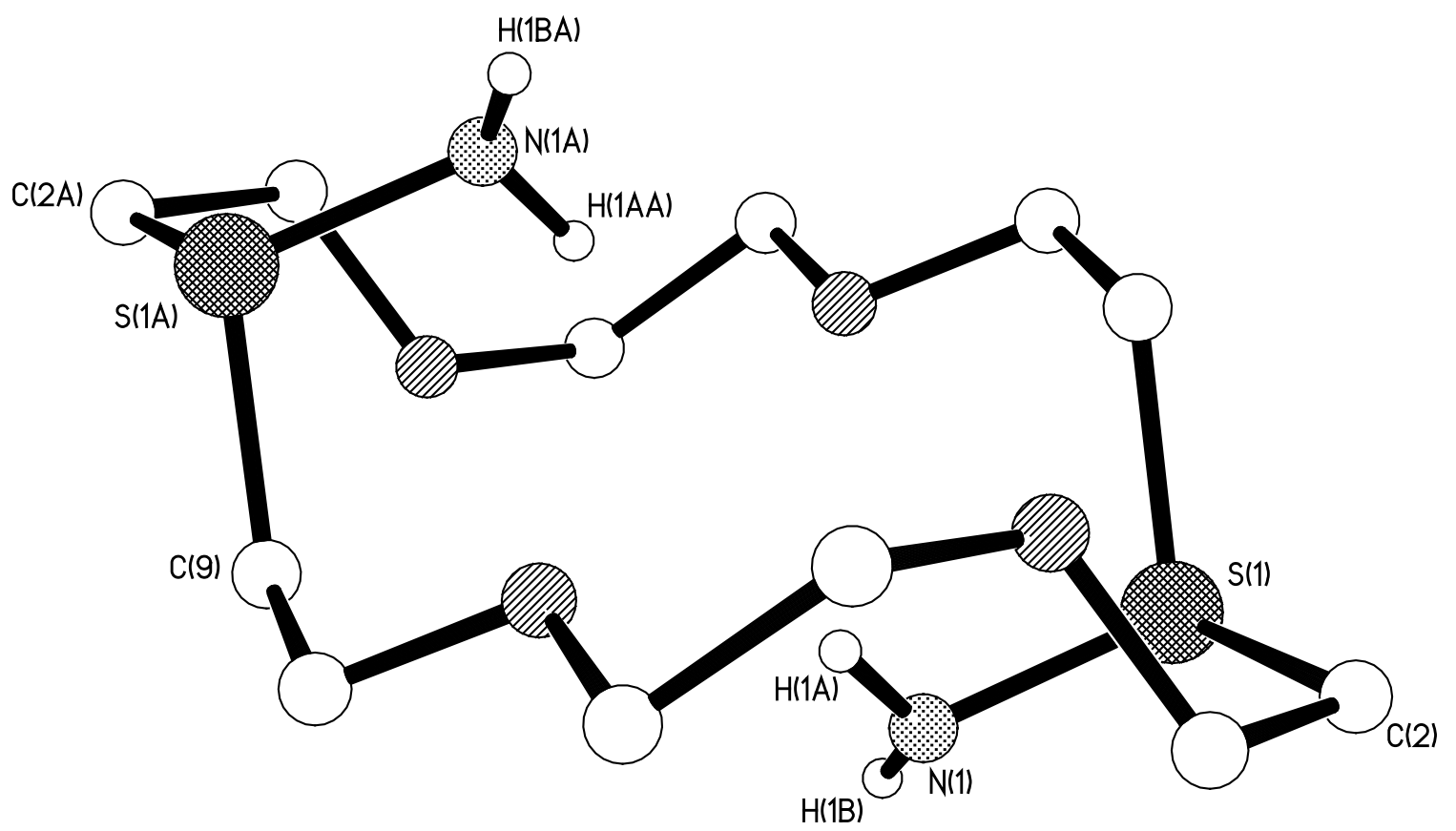

${ }_{20}$ Fig.5 X-ray crystal structure of the dication within $[3 \mathbf{b}]\left[\mathrm{BPh}_{4}\right]_{2}$. 


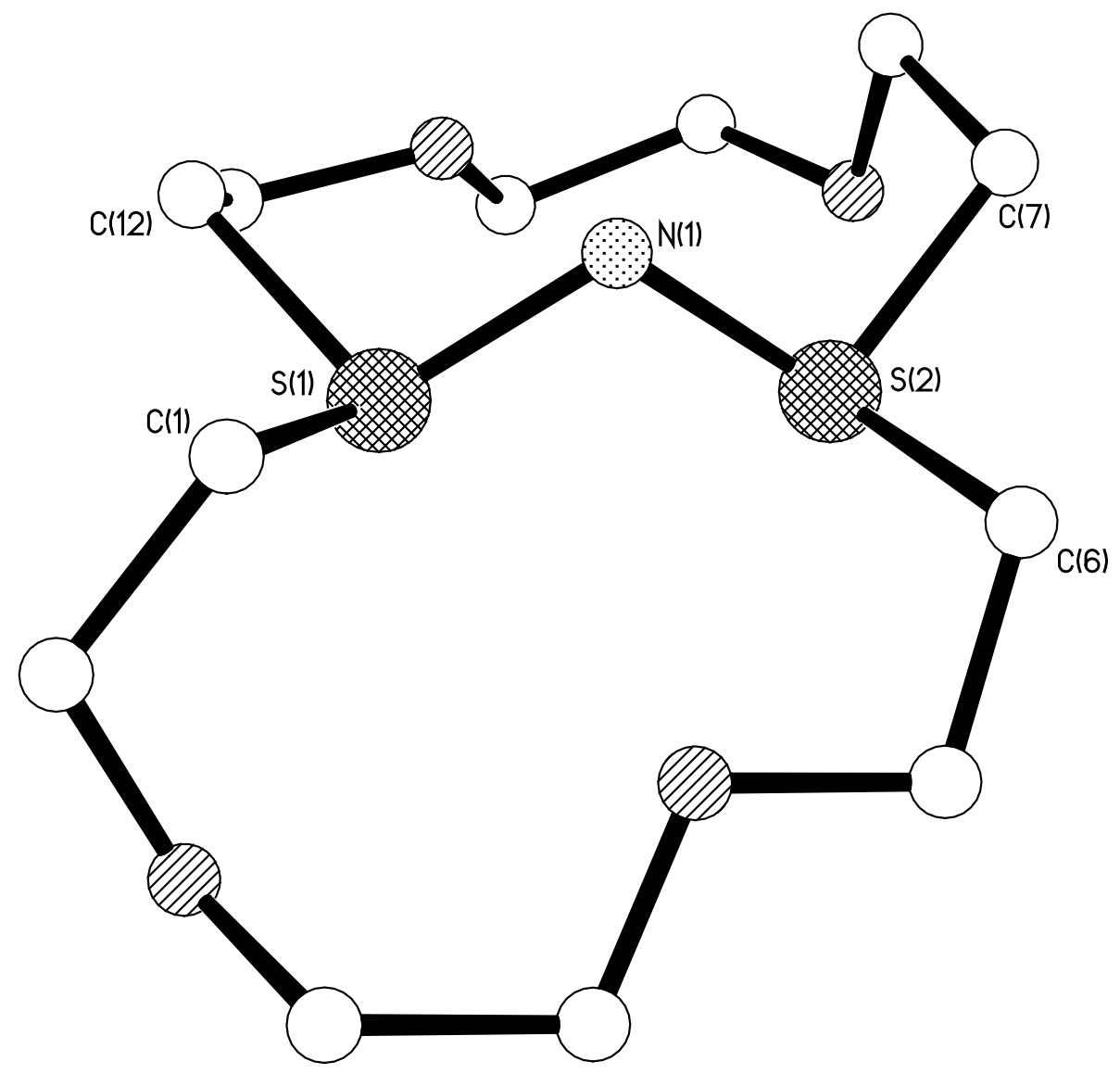

Fig.6 The X-ray crystal structure of one of the independent cations in [6][ $\left.\mathrm{BPh}_{4}\right]$. 
Table 1. Crystallographic parameters ${ }^{a}$

\begin{tabular}{|c|c|c|c|c|c|}
\hline Compound & {$[2 \mathrm{a}]\left[\mathrm{BPh}_{4}\right]$} & {$[4]\left[\mathrm{BPh}_{4}\right]$} & {$[5]\left[\mathrm{BPh}_{4}\right]$} & {$[3 \mathrm{~b}]\left[\mathrm{BPh}_{4}\right]_{2}$} & {$[6]\left[\mathrm{BPh}_{4}\right]$} \\
\hline \multirow[t]{2}{*}{ Formula } & $\mathrm{C}_{32} \mathrm{H}_{38} \mathrm{BNO}_{3} \mathrm{~S}$ & $\mathrm{C}_{36} \mathrm{H}_{44} \mathrm{BNO}_{4} \mathrm{~S}_{2}$ & $\mathrm{C}_{41} \mathrm{H}_{53} \mathrm{BCl}_{3} \mathrm{NO}_{6} \mathrm{~S}_{2}$ & $\mathrm{C}_{60} \mathrm{H}_{68} \mathrm{~B}_{2} \mathrm{~N}_{2} \mathrm{O}_{4} \mathrm{~S}_{2}$ & $\mathrm{C}_{36} \mathrm{H}_{44} \mathrm{BNO}_{4} \mathrm{~S}_{2}$ \\
\hline & 527.50 & 629.65 & 837.12 & 966.90 & 629.65 \\
\hline \multicolumn{6}{|l|}{ M } \\
\hline Crystal dimensions & $0.23 \times 0.21 \times 0.13$ & $0.15 \times 0.15 \times 0.15$ & $\begin{array}{l}0.13 \times 0.03 \times \\
0.03\end{array}$ & $0.10 \times 0.10 \times 0.08$ & $0.65 \times 0.32 \times 0.12$ \\
\hline $\begin{array}{l}\text { Crystal morphology and } \\
\text { colour }\end{array}$ & Block, colourless & Prism, colourless & $\begin{array}{l}\text { Needle, } \\
\text { colourless }\end{array}$ & Prism, colourless & Block, colourless \\
\hline Crystal system & Monoclinic & Monoclinic & Monoclinic & Monoclinic & Triclinic, \\
\hline Space Group & $P 2_{1} / n$ & $P 2_{1} / c$ & $P 2_{1} / C$ & $P 2_{1} / c$ & $P \overline{1}$ \\
\hline$a / \AA$ & $12.9446(3)$ & $10.7110(11)$ & 14.046(3) & $12.854(3)$ & $10.6347(5)$ \\
\hline$b / \AA$ & $12.0724(3)$ & $27.872(2)$ & $11.934(2)$ & $12.341(3)$ & $14.3378(7)$ \\
\hline$c / \AA ̊$ & $18.1166(5)$ & $11.0118(12)$ & $25.163(6)$ & $16.969(4)$ & $22.9147(11)$ \\
\hline$\alpha /^{\circ}$ & 90 & 90 & 90 & 90 & $78.764(2)$ \\
\hline$\beta /^{\circ}$ & $98.060(4)$ & 101.127(3) & $98.981(6)$ & 108.861(10) & $81.805(2)$ \\
\hline$y I^{\circ}$ & 90 & 90 & 90 & 90 & $71.051(2)$ \\
\hline \multirow[t]{2}{*}{$V I \AA^{3}$} & 2803.16(18) & $3225.6(6)$ & $4166.5(16)$ & 2547.2(11) & $3229.5(3)$ \\
\hline & 4 & 4 & 4 & 2 & 4 \\
\hline \multicolumn{6}{|l|}{ Z } \\
\hline$\mu / \mathrm{mm}^{-1}$ & 0.149 & 0.206 & 0.367 & 0.155 & 0.206 \\
\hline$\theta$ Range $^{\circ}$ & 1.82 to 30.56 & 2.02 to 25.35 & 1.89 to 25.38 & 2.54 to 25.35 & 0.91 to 28.90 \\
\hline Measured reflections & 32677 & 20502 & 22437 & 14321 & 28453 \\
\hline Independent reflections & 8548 & 5821 & 6919 & 4600 & 14816 \\
\hline$R_{\text {int }}$ & 0.0260 & 0.0278 & 0.0567 & 0.1030 & 0.0238 \\
\hline$R\left[F^{2}>2 \sigma\left(F^{2}\right)\right]^{a}$ & 0.0438 & 0.0375 & 0.1066 & 0.0849 & 0.0464 \\
\hline$w R 2$ [all data] & 0.1206 & 0.0927 & 0.2964 & 0.2254 & 0.1268 \\
\hline $\begin{array}{l}\text { Largest difference map } \\
\text { features/e } \AA^{-3}\end{array}$ & $0.524,-0.303$ & $0.294,-0.315$ & $0.889,-0.846$ & $1.132,-0.361$ & $0.570,-0.349$ \\
\hline
\end{tabular}

